\title{
Aire en el espacio periportal: Más allá de la clásica tríada
}

\author{
Drs. Leonardo Lidid $A^{(1)}$, Sebastián Yévenes $A^{(2)}$, Fabiola Vargas $P^{(2)}$.
}

1 Profesor Asistente de Radiología. Campus Occidente, Facultad de Medicina, Universidad de Chile. Hospital San Juan de Dios. Staff Hospital Dipreca.

2 Residente de Radiología. Campus Occidente, Facultad de Medicina, Universidad de Chile, Hospital San Juan de Dios.

\section{Air in periportal space: Beyond the classic triad}

\begin{abstract}
The intrahepatic air, in particular gas within the portal venous or within biliary system, exhibits typical morphological patterns clearly seen on CT studies. Nevertheless, this gas can also be found in the periportal space, a little-known extension of the subperitoneal space, showing intrahepatic distribution patterns similar to those described above, but with completely different pathological and diagnostic implications. This fact led us to characterize this sign and its findings on CT scans, along with its main differential diagnoses.

Keywords: Bile ducts, Cystoides intestinalis, Intestinal perforation, Pneumatosis, Pneumoperitoneum, Portal system, Retroperitoneal space.
\end{abstract}

Resumen: El gas intrahepático, en particular el ubicado en relación con la vía biliar y la porta, tiene patrones morfológicos característicos especialmente evidentes en los estudios por tomografía computada. El aire,sin embargo, no solamente puede encontrarse en estos lugares, sino que también puede ubicarse en el espacio periportal; una extensión poco conocida del espacio subperitoneal, con patrones de distribución intrahepáticos similares a los ya descritos, pero con implicancias patológicas y diagnósticas completamente diferentes. Por estas razones se decide caracterizar este signo y sus hallazgos en TAC, así como sus principales diagnósticos diferenciales.

Palabras clave: Cystoides intestinalis, Ductos Biliares, Espacio Retroperitoneal, Perforación intestinal, Pneumatosis, Pneumoperitoneo, Sistema portal.

Lidid L y cols. Aire en el espacio periportal: Más allá de la clásica tríada. Rev Chil Radiol 2011; 17(3): 120-125. Correspondencia: Dr. Leonardo Lidid A.

Dirección postal: Facultad de Medicina. Campus Occidente. Universidad de Chile. Casilla 33052-correo 33. Santiago leolidid@gmail.com

Trabajo recibido el 03 de junio de 2011, aceptado para publicación el 23 de junio de 2011.

\section{Introducción}

El aire en el cuerpo humano puede tener muchas distribuciones, algunas de ellas normales y otras patológicas. En estas últimas, el gas intrahepático, en particular el ubicado en relación a la vía biliar y la porta, tienen un patrón de distribución característico, especialmente evidente en los estudios por tomografía computada. A pesar de ello, el aire también puede tener una distribución muy similar y, sin embargo, no estar directamente vinculado a lo que habitualmente se conoce como tríada portal. De algo nos estamos olvidando. Lo que está alrededor. El aire y otros procesos patológicos, pueden afectar el espacio periportal, lo que implica una gamma de diagnósticos diferenciales importantes, habitualmente ignorados al momento de evaluar un estudio tomográfico. En particular, el desconocimiento en esta área, pareciera tener una mayor relevancia cuando se trabaja en sistemas de atención de urgencia, en especial en pacientes con patología abdominal aguda. Por estas razones decidimos hacer una descripción de este espacio, con el significado de la presencia de aire a este nivel, sus principales diagnósticos diferenciales y su aspecto morfológico en estudios por tomografía axial computada. 


\section{Espacio periportal}

La cápsula de Glisson cubre la superficie hepática como una extensión del peritoneo, excepto por el área desnuda. La vaina de Glisson corresponde a la parte de la cápsula que rodea el sistema porta y se extiende dentro del espesor del hígado (Figura 1A).
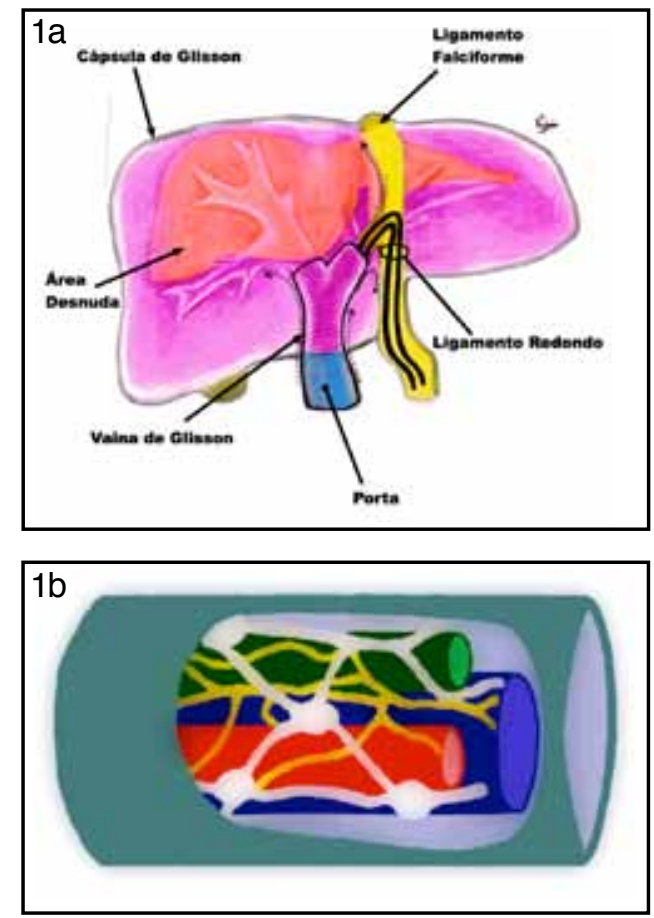

Figura 1. a) Cápsula y vaina de Glisson. El diagrama muestra la continuidad de la vaina de Glisson, que se extiende rodeando la porta y su prolongación a través del ligamento teres-falciforme (ver figura 3). b) Espacio periportal. El diagrama muestra la vena porta (azul) y las estructuras de la región periportal incluyendo la arteria hepática (rojo), vía biliar (verde), linfáticos (blanco) y nervios (amarillo). El tejido intersticial conectivo no se representa, pero corresponde al espacio que existe entre todas estas estructuras.

La tríada portal, los nervios y los linfáticos (Figura 1b) siguen este camino de tejido conectivo a través del hígado, constituyéndose esta zona, en una extensión del espacio subperitoneal (subseroso) que viene desde el ligamento gastrohepático y hepatoduodenal (Figura 2a-b) $^{(1,2,3)}$.

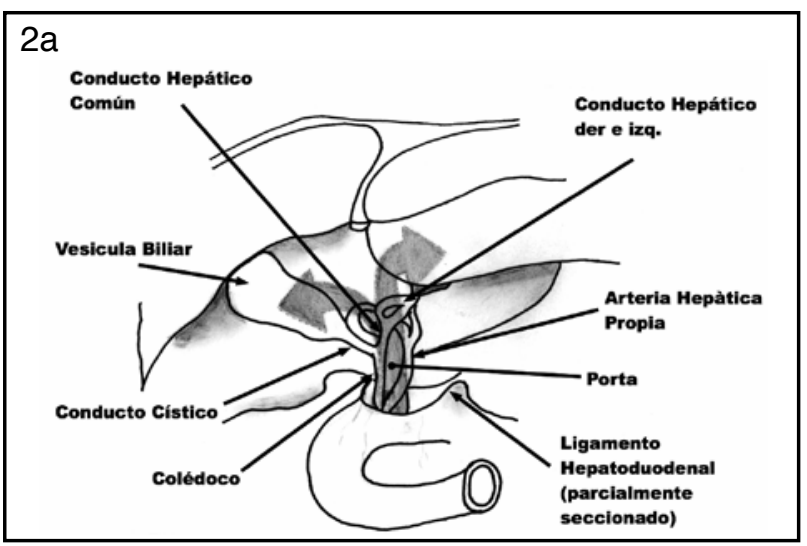

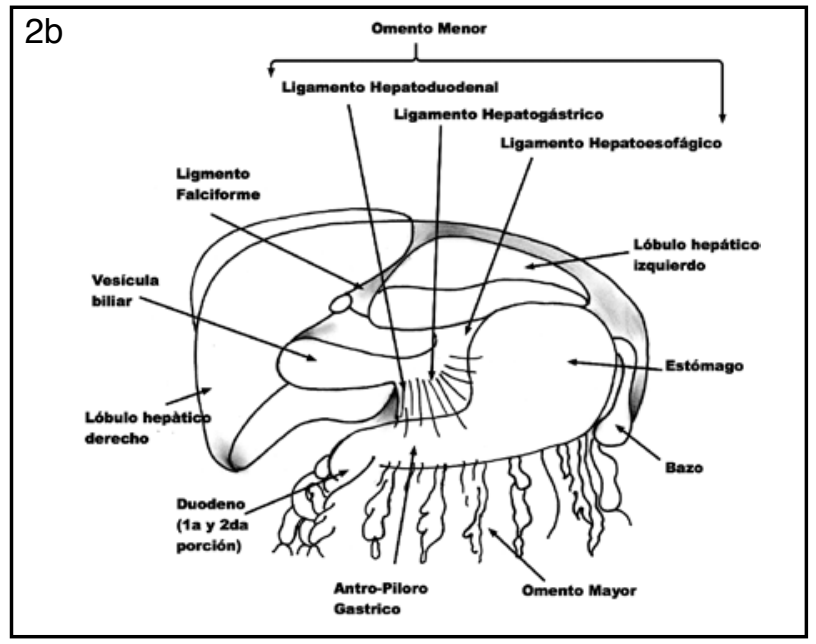

Figura 2. a) Relación anatómica de la tríada portal, antro gástrico y bulbo duodenal. El antro gástrico y bulbo duodenal están ligados al omento menor. b) El hígado y los distintos segmentos del tracto gastrointestinal están interconectados a través de ligamentos y mesenterios. Entre hígado y estómago existen los ligamentos gastrohepático y el ligamento hepatoduodenal. El espacio subperitoneal es la base de la potencial interconexión entre retroperitoneo y la cavidad peritoneal. Este espacio de interconexión complejo está compuesto principalmente de tejido graso que contiene estructuras nerviosas, vasos y tejido linfático envueltos por capas serosas que constituyen los ligamentos y mesenterios asociados a los principales órganos y vísceras abdominales. Por lo tanto, representa un canal importante para la propagación de enfermedades dentro de la cavidad peritoneal. La flecha gruesa en 2a) representa las posibles vías de diseminación del aire, entre las hojas del omento menor, disecando el espacio periportal.

La vaina de Glisson, a nivel hepático, también se continúa hacia cefálico con el espacio subperitoneal del ligamento teres y el ligamento falciforme (Figura $1 \mathrm{a}$ ).

De esta forma la continuidad anatómica del espacio subperitoneal permite la diseminación de enfermedades, no sólo entre las estructuras intraperitoneales, sino que también entre las regiones extra e intraperitoneales ${ }^{(1,4,5)}$.

Dentro de los fenómenos patológicos que pueden ocupar la vía periportal de diseminación se incluye líquido (edema), sangre, procesos inflamatorios (exudados pancreáticos), infiltraciones tumorales (Figura 3) y aire ${ }^{(1,2,3)}$.

Este último se asocia frecuentemente, aunque no únicamente, a perforación de víscera hueca, principalmente del segmento gastrointestinal alto (especialmente si es reciente) pudiendo observarse gas intrahepático en ubicación periportal y por extensión en la fisura hepática rodeando el ligamento redondo (Figura 4), continuándose por esta vía, incluso hasta su inserción en el ombligo ${ }^{(1,3,6)}$. 



Figura 3. Paciente con Carcinomatosis peritoneal de primario desconocido con invasión del espacio periportal y ligamento teres. Cortes progresivamente más cefálicos. a-b) Se observa una masa carcinomatosa que compromete el interior del saco menor (cabezas de flecha), ventral al páncreas (flechas anchas) y que en c) invade el hilio hepático, comprometiendo el espacio periportal (flechas negras), comunicándose a través de éste con el ligamento teres (flechas blancas), que se observa mucho mejor en a y b). Metástasis hepática (*).
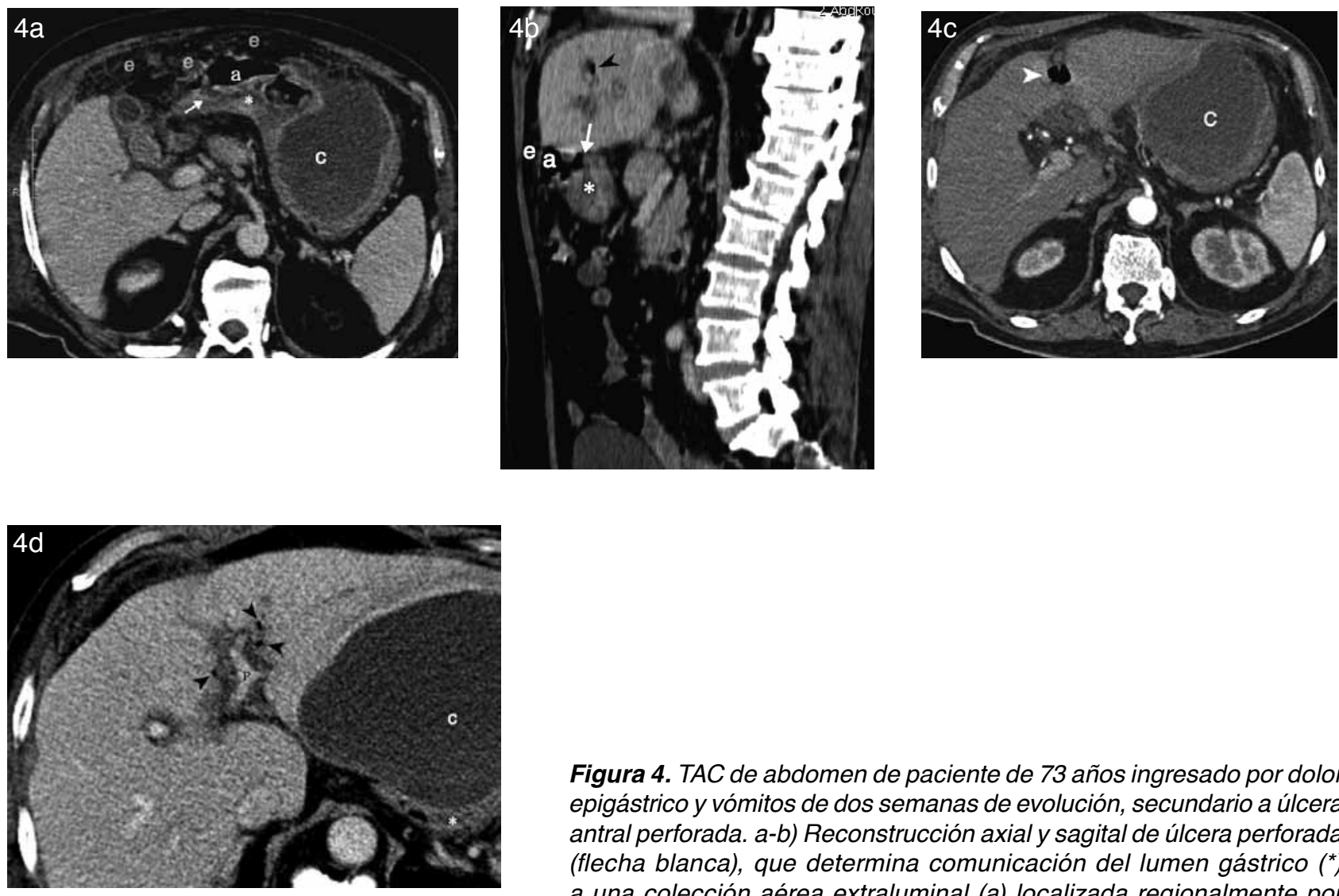

Figura 4. TAC de abdomen de paciente de 73 años ingresado por dolor epigástrico y vómitos de dos semanas de evolución, secundario a úlcera antral perforada. a-b) Reconstrucción axial y sagital de úlcera perforada (flecha blanca), que determina comunicación del lumen gástrico ( ${ }^{*}$ ) a una colección aérea extraluminal (a) localizada regionalmente por epiplón aplastronado (e). También se ve una colección líquida (c) en el espacio subseroso del epiplón menor (curvatura mayor) secundario a la perforación ya descrita. $c$-d) Reconstrucciones axiales que muestran la extensión subperitoneal del gas, observándose tanto a nivel del ligamento redondo (cabeza de flecha blanca), como en relación al espacio intersticial periportal engrosado [edema periportal] (cabezas de flecha negras). Originalmente el aire periportal se interpretó como aire biliar, sin embargo la colangiografía intraoperatoria (no mostrada) lo descarta. e) Detalle del lóbulo hepático izquierdo que muestra una rama de la porta izquierda (p), flanqueada por dos hipodensidades, observándose el intersticio periportal edematizado y disecado parcialmente por aire (cabeza de flecha). 
El gas, en este caso, se ubica en el tejido intersticial que rodea la porta siguiendo su distribución tanto intra como extrahepática (Figura 5). Sin embargo, la vía biliar y la porta en sí mismas pueden, en determinadas condiciones, contener aire y asemejar la distribución de los hallazgos antes descritos, especialmente cuando éste es de baja cuantía.
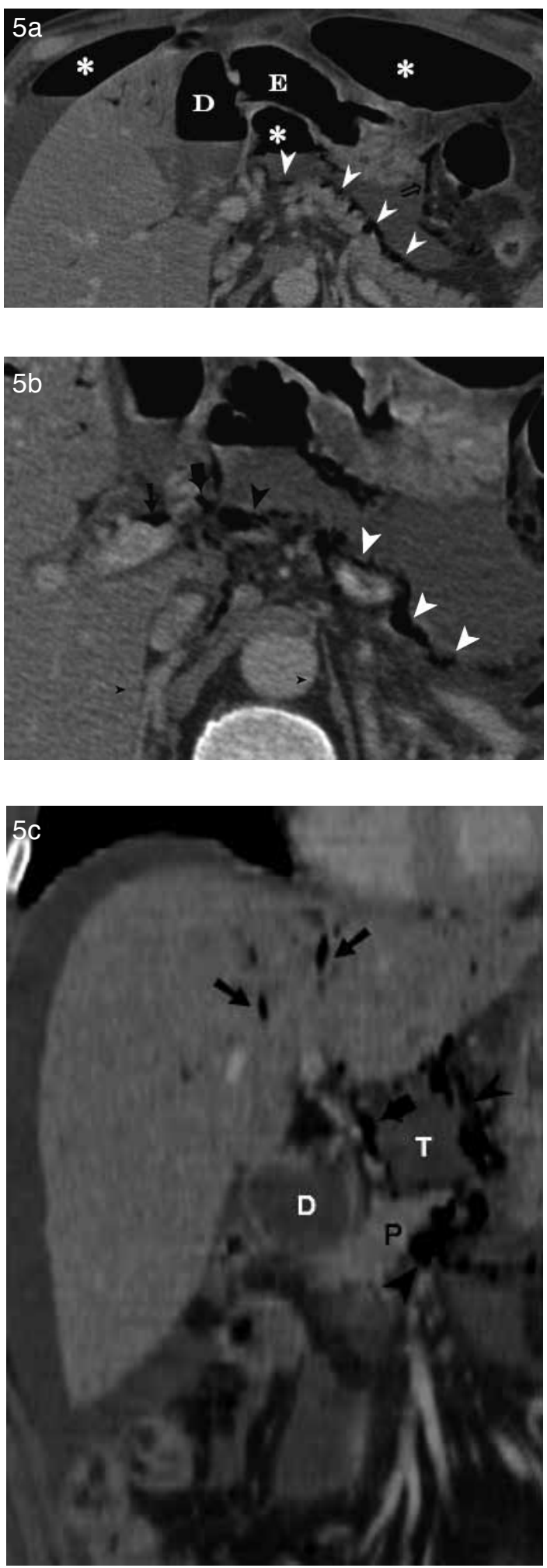

\section{Gas Portal}

El gas porto-mesentérico y su hallazgo asociado, la neumatosis intestinal, son signos que tradicionalmente se han vinculado a un pronóstico ominoso, ya que generalmente indican una injuria intestinal aguda, habitualmente isquémica o infecciosa. Sin embargo, cualquier cosa que altere la pared intestinal y produzca una disrupción de la superficie mucosa puede producir estos hallazgos, incluyéndose dilatación patológica de asas, ulceraciones intestinales, trauma, enfermedad inflamatoria intestinal y procedimientos quirúrgicos entre otros. De este modo también hay una serie de diagnósticos benignos que se pueden asociar a esta signología, por lo que es importante considerar en cada caso, el contexto clínico del paciente ${ }^{(7,8)}$.

En tomografía computada, se pueden observar imágenes tubulares, de densidad aérea que se ramifican desde el centro hacia la periferia hepática, llegando incluso a contactar la cápsula de este órgano (Figura 6 $a-b)^{(8,9,10)}$. El aire entonces, dependiendo de la cuantía, tiende a tener una distribución antigravitatoria con predominancia en el lóbulo hepático izquierdo. Los hallazgos asociados, como la neumatosis intestinal, también se pueden apreciar (Figura $6 \mathrm{c}-\mathrm{d})^{(7)}$.

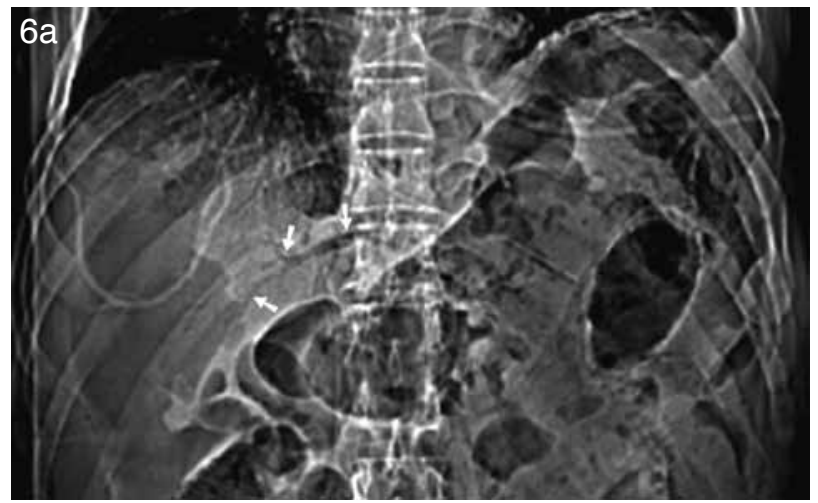

Figura 5. Paciente de sexo masculino de 48 años de edad con isquemia mesentérica aire portal y subsecuente perforación de víscera hueca, con aire subperitoneal y severo neumoperitoneo secundario. a-b) Cortes axiales de TC que muestran extensión de gas por vía subperitoneal adyacente al páncreas, a la vena esplénica (cabezas de flecha blanca) y a través del ligamento gastroesplénico (flecha de doble barrilete). También hay extensión cefálica de aire por el ligamento hepatoduodenal, adyacente a la arteria hepática común (cabeza flecha negra) y propia (flecha gruesa). También hay gas intraportal (flecha) secundario a isquemia. c) Reconstrucción coronal que representa mejor la extensión del gas vía ligamento hepatoduodenal (flecha gruesa) (compárese con la Figura 2a). El gas no alcanzó a disecar el intersticio intrahepático, lo que se observa es aire portal (flechas). También hay gas omental y retroperitoneal (cabezas de flecha). Cabeza de páncreas (p). Líquido libre y neumoperitoneo(*). Duodeno (d). Estómago (e). Transcavidad de los epiplones (t). 

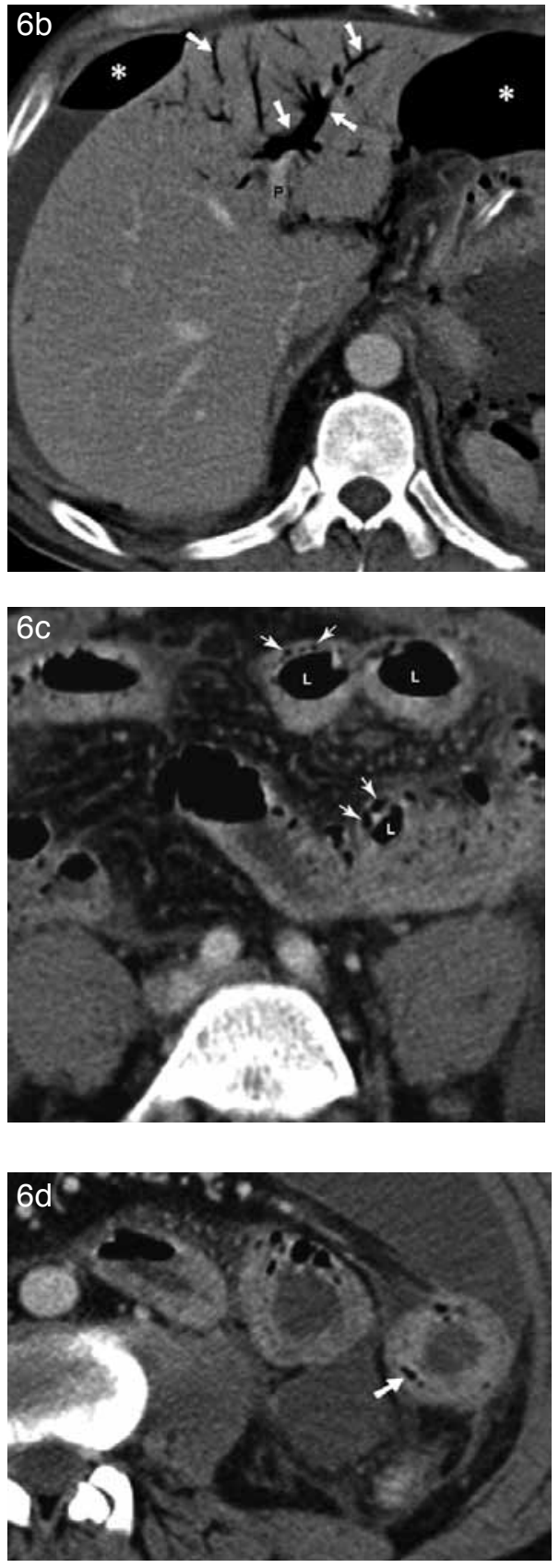

Figura 6. Aire portal. Mismo paciente que en Figura 5. a) Scout View que muestra neumoperitoneo y aire portal. Es difícil definir realmente si el gas está ubicado en la porta (flechas blancas). En general, se describe, que la radiografía es relativamente inespecífica para realizar este diagnóstico, porque suele confundirse con aerobilia. La ubicación del gas es más fácil de determinar en los cortes axiales. b). Corte axial de tomografía computada que muestra la porta (p) con aire (flechas blancas) que llega a contactar la cápsula del lóbulo hepático izquierdo, neumoperitoneo y líquido libre $\left({ }^{*}\right)$. c) Cortes axiales de TC a nivel más caudal, donde se ve dilatación patológica de asas, con engrosamiento parietal y neumatosis. Nótese como el gas intramural (flechas), está separado del lumen (L) intestinal, lo que se hace más evidente en d) por la posición antigravitatoria de éste (flecha). También se observa líquido libre subfrénico e interasas.
Gas en la vía biliar (Aerobilia)

Se refiere a la acumulación de aire en el árbol biliar secundario a múltiples causas, siendo las más frecuentes las anastomosis bilioentéricas quirúrgicas, las fístulas bilioentéricas espontáneas y la incompetencia del esfínter de Oddi (comúnmente iatrogénica, secundarias por ejemplo a esfinterotomía post ERCP y más raramente no iatrogénicas, por ejemplo secundaria al paso reciente de cálculos, entre otras). Causas menos frecuentes incluyen las infecciosas (Colangitis), y las fístulas broncopleurales ${ }^{(9)}$.

En tomografía computada, el gas tiene una distribución característica en el scout view (Figura 7 a) que se correlaciona perfectamente con su morfología en los cortes axiales, donde se visualiza como ramificaciones con densidad de aire que tienden a tener una ubicación más central, confluyendo en el conducto hepático común (por el flujo centrípeto de la bilis) (Figura 7 b-c), con preferencia, tal como fue descrito respecto al aire en la porta, en la ocupación del lóbulo hepático izquierdo. El aire en la periferia, por la disposición del flujo biliar habitualmente no llega más allá de $2 \mathrm{~cm}$ de la cápsula hepática (Figura $7 d)^{(9,11,12)}$.
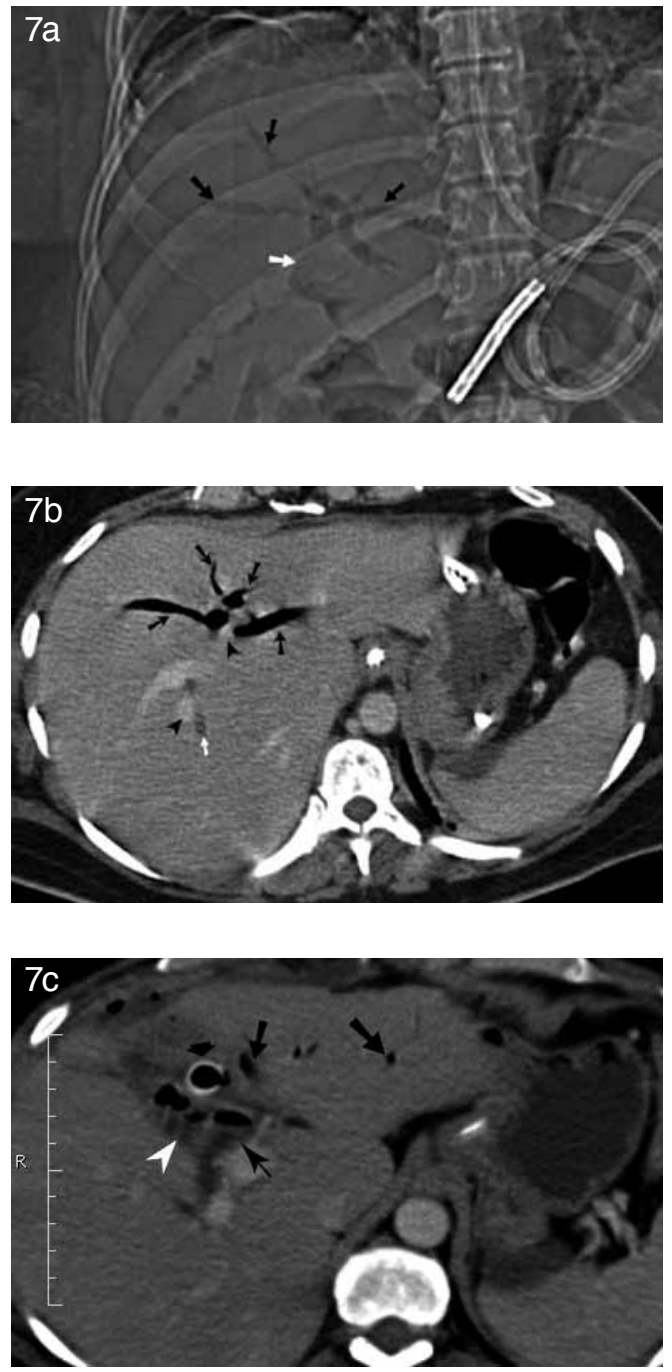


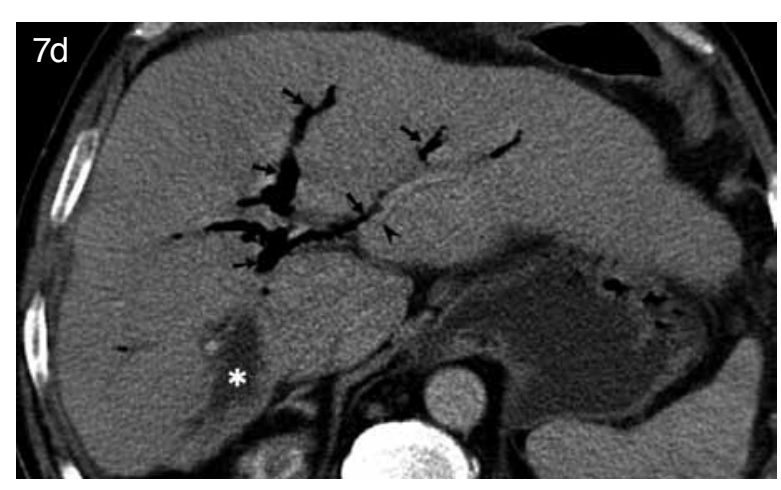

Figura 7. Mujer de 45 años con derivación bilio-digestiva por pancreatitis recurrente secundaria a variante anatómica de la unión colédoco-pancreática. a) Scout View. Clásica imagen radiológica en forma de " $Y$ " en el cuadrante superior derecho del abdomen, correspondiente a los conductos biliares común derecho e izquierdo. Vía biliar intrahepática con aire (flechas negras) y hepático común con aire (flecha blanca) justo antes de su anastomosis distal. b) Corte axial de TC contrastada donde se ve el aire biliar llegando hasta 2 cm antes de la cápsula hepática (flechas negras). La vía biliar del aspecto posterior (flecha blanca), si bien está dilatada no tiene aire en su lumen (distribución antigraviatoria). Porta (cabeza de flecha). c) Corte axial de TC donde se observa el asa ascendida (cabeza flecha) anastomosada con el hepático común (flecha fina). También se alcanza a ver aire biliar (flechas negras), y un drenaje en el sitio quirúrgico (flecha ancha). d) Corte axial de otro paciente en que se observa mejor la ubicación del aire en el trayecto biliar (flechas negras), separado de la porta (cabeza de flecha). Incidentalmente se aprecia daño hepático crónico y dilatación focal secuelar de la vía biliar regional posterior sin aire $\left(^{*}\right)$.

\section{Conclusiones}

El espacio periportal es poco conocido como una región de compromiso de patología abdominal. Este espacio es mucho mejor comprendido si se considera como una extensión intrahepática del espacio subperitoneal (mesentérico y retroperitoneal). Aunque cualquier proceso con diseminación subperitoneal puede afectarlo, la presencia de aire a este nivel es muy sugerente de perforación de víscera hueca, principalmente del segmento gastrointestinal alto, debiendo distinguirlo de sus principales diagnósticos diferenciales: el aire portal y biliar, ya que su etiología y pronóstico clínico son completamente distintos.

\section{Bibliografía}

1. Kim S, Kim TU, Lee JW, Lee TH, Lee SH, Jeon TY, et al. The perihepatic space: comprehensive anatomy and CT features of pathologic conditions. Radiographics. 2007; 27(1): 129-143.

2. Karcaaltincaba M, Haliloglu M, Akpinar E, Akata D, Ozmen M, Ariyurek M, et al. Multidetector CT and MRI findings in periportal space pathologies. Eur J Radiol. 2007; 61(1): 3-10. Epub 2006 Nov 22.

3. Cho HS, Yoon SE, Park SH, Kim H, Lee YH, Yoon KH. Distinction between upper and lower gastrointestinal perforation: Usefulness of the periportal free air sign on computed tomography. Eur J Radiol. 2009; 69(1): 108-113.

4. Silverman PM. The subperitoneal space: mechanisms of tumour spread in the peritoneal cavity, mesentery, and omentum. Cancer Imaging. 2003; 4(1): 25-29.

5. Oliphant M, Berne AS, Meyers MA. The subperitoneal space of the abdomen and pelvis: planes of continuity. AJR Am J Roentgenol. 1996; 167(6): 1433-1439.

6. Meyers MA. The extraperitoneal space: normal and pathologic anatomy. In: Meyers MA, ed. Dynamic radiology of the abdomen. 5th ed. New York, NY: Springer-Verlag 2000; 333-492.

7. Sebastià $C$, Quiroga $S$, Espin E, Boyé R, AlvarezCastells A, Armengol M. Portomesenteric vein gas: pathologic mechanisms, CT findings, and prognosis. Radiographics. 2000; 20(5): 1213-1224; discussion 1224-1226.

8. Hussain A, Mahmood H, El-Hasani S. Portal vein gas in emergency surgery. World J Emerg Surg. 2008; 3: 21

9. Goldstein WB, Cusmano JV, Gallagher JJ, Hemley $\mathrm{S}$. Portal vein gas. A case report with survival. Am J Roentgenol Radium Ther Nucl Med. 1966; 97(1): 220222.

10. Neumatosis intestinal y gas portomesentérico: a propósito de un caso. Alonso-Burgos A, Nogueral JJ, Cosín O, Viudez A, Pueyo J, Elorz M, et al. Rev Med Univ Navarra 2007; 51(2): 3-6.

11. Sherman SC, Tran H. Pneumobilia: benign or lifethreatening. J Emerg Med 2006; 30 (2): 147-153.

12. Lewandowski BJ, Withers $C$, Winsberg F. The Air-Filled Left Hepatic Duct: The Saber Sign as an Aid to the Radiographic Diagnosis of Pneumobilia. Radiology 1984; 153 (2): 329-332. 